\title{
Preparation and physicochemical evaluation of emulsified virgin coconut oil (VCO)- Carica papaya extract concoction using Tween80
}

\begin{abstract}
Carica papaya is a member of the Caricaceae. Its leaves have been used in folk medicine for centuries. Recent studies have shown its beneficial effects as an anti-inflammatory agent (Owoyele et al 2008) and anti-tumour15 as well as antioxidant and wound healing properties7. The study has shown that the effect of carica papaya leaves juice intake also can accelerate the rate of increase in platelet count among the patients infected with dengue fever and dengue haemorrhagic fever18. With all the goodness of carica papaya leaves, a formulation with addition of virgin coconut oil (VCO) is produced to give an enhanced supplement beverage to market nowadays. Virgin coconut oil is well known as anti-oxidant4. The combination of these two substances gives a balance combination in healthy supplement. In recent years the application of emulsion is rapidly increasing in many fields such as cosmetics and paints. Emulsions are dispersions of droplets of one liquid in another, immiscible, liquid in which the droplets are of colloidal or near-colloidal sizes. The combination of water and oil ( $\mathrm{VCO}$ ) with addition of non-ionic surfactant Tween80 was constructed using ternary phase diagram. By considering the Hydrophilic-Lipophilic Balance (HLB) value of each substance will help in producing a stable emulsion.
\end{abstract}

Keyword: Carica papaya; Emulsion; Oil-in-water; Ternary phase diagram 